\title{
APLICAÇÃO DE REGULADORES VEGETAIS NO RETARDAMENTO DA ABSCISÃO DE FRUTOS DE LARANJEIRA-'HAMLIN'1
}

\author{
ISOLINA MARIA LEITE DE ALMEIDA², JOÃO DOMINGOS RODRIGUES ${ }^{3}$, \\ ELIZABETH ORIKA ONO ${ }^{3}$
}

\begin{abstract}
RESUMO - O trabalho avaliou os efeitos de auxinas e giberelina, combinadas e aplicadas em pré-colheita no desenvolvimento e na porcentagem de queda natural dos frutos de laranjeira- 'Hamlin' (Citrus sinensis Osbeck). Foram realizadas 3 aplicações a intervalos de 45 dias dos seguintes tratamentos, via foliar: GA + NAA a 12,5mg.L-1 de cada; GA + NAA a 25mg.L $\mathrm{L}_{3}^{-1} ; \mathrm{GA}_{3}+2,4-\mathrm{D}$ a $12,5 \mathrm{mg} . \mathrm{L}^{-1} ; \mathrm{GA}_{3}$

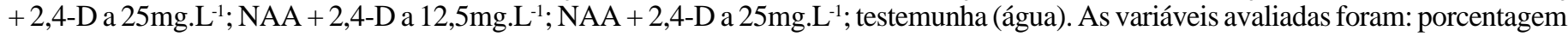
de queda natural dos frutos $(\%)$, massa fresca de frutos $(\mathrm{g})$ e teor de suco no fruto (\%). Os resultados obtidos mostram que a utilização de reguladores vegetais atrasa a queda natural de frutos de laranjeira- 'Hamlin', podendo-se prolongar o período de colheita, destacando-se o tratamento com GA $325 \mathrm{mg} . \mathrm{L}^{-1}+2,4-\mathrm{D} 25 \mathrm{mg}$. $\mathrm{L}^{-1}$ e também não influenciaram no aumento da massa fresca e no teor de suco dos frutos.
\end{abstract}

Termos de indexação: massa fresca, teor de suco, auxinas, giberelina

\section{PLANT GROWTH REGULATORS AS DELAYING FACTORS TO THE ABSCISSION OF THE ORANGE FRUIT}

\begin{abstract}
This research evaluated the effects of auxins and gibberellin, combined and applied preharvest in the development of the orange cv. Hamlin tree fruit and natural fall percentage. The orange trees (Citrus sinensis Osbeck) cv. Hamlin were grafted in lemon 'Cravo' (Citrus limonia Osbeck). The treatments used were made in 3 applications with intervals of 45 days: GA $+\mathrm{NAA}_{3}$ at 12.5mg.L ${ }^{-1}$;

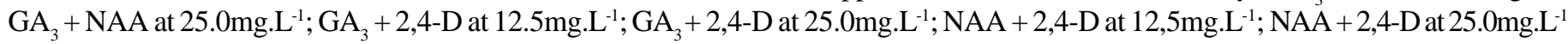
and control (water). The parameters evaluateds were: natural fruit fall percentage, fresh fruit weight ( $\mathrm{g}$ ) and juice content $(\%)$. The results showed that the treatment with $\mathrm{GA}_{3}+2,4-\mathrm{D} 25.0 \mathrm{mg} . \mathrm{L}^{-1}$ provided a reduction in the natural fruit fall percentage compared to the control, thus extending the harvest time. The treatments didn't increase the fruit weight and juice content.
\end{abstract}

Index terms: Citrus sinensis, bioregulators, fruit drop

\section{INTRODUÇÃO}

No Brasil, a safra de citros tem início no mês de abril, com a colheita de cultivares precoces, como a 'Hamlin'. Nos meses de setembro a outubro, colhe-se, especialmente, a cultivar 'Pêra' e termina-se com os tardios, como 'Natal'e 'Valência', de novembro a janeiro. Nota-se que, nos meses de julho a agosto, a indústria cítrica sofre redução de suas atividades com o final da colheita das cultivares precoces, aguardando-se o início da colheita da cultivar Pêra.

Necessita-se, dessa forma, da introdução de tecnologias que consigam atrasar parte da safra da cultivar Hamlin, diminuindo-se, assim, tais problemas de planejamento e proporcionando a melhoria da qualidade de nossa fruta cítrica para o mercado externo.

Monselise \& Goren (1978) relatam que o uso de 2,4-D na prevenção da queda pré-colheita em citros foi o primeiro tratamento desenvolvido e mundialmente usado. Os mesmos autores descrevem que pulverizações de auxinas previnem a queda dos frutos pela manutenção das células da zona de abscisão. $\mathrm{O}$ ba- lanço hormonal previne a síntese de enzimas hidrolíticas, como a celulase, que degrada a parede celular e atua na atividade da poligalacturonase, que, juntamente com a celulase, seriam as responsáveis pela degradação dos principais componentes da parede celular, a celulose e a pectina. Outra auxina sintética, o ácido naftaleno-acético (NAA), também apresenta efeito semelhante ao 2,4-D, como o relatado por vários autores como Patil et al. (1989) e Llanos et al. (1991).

Outro regulador vegetal muito utilizado na citricultura é o ácido giberélico, que atrasa a diminuição do teor de clorofila na casca, reduzindo o acúmulo de carotenóides (Ragone, 1992) e, dessa forma, atrasando a senescência da casca. Chitarra \& Chitarra (1990) relatam que as auxinas e giberelinas têm sido estudadas por funcionarem, total ou parcialmente, como retardadores da senescência de frutos cítricos. As auxinas endógenas atuam inibindo a síntese de etileno, retardando o amadurecimento e mantendo os tecidos em seu estado juvenil, sem afetar no amadurecimento posterior. As giberelinas afetam na mudança de cor, uma vez que retardam a perda de clorofila, o acúmulo de carotenóides e o amaciamento da casca.

1 (Trabalho 159/2001). Recebido: 27/08/2001. Aceito para publicação: 11/03/2002.

2 Eng Agr $^{\mathrm{a}}$ - M.Sc. - Doutora em Agronomia - AC: Horticultura, Faculdade de Ciências Agronômicas, Campus de Botucatu, Universidade Estadual Paulista - UNESP.

3 Departamento de Botânica, Instituto de Biociências, Campus de Botucatu, Universidade Estadual Paulista - UNESP, C.P. 510, CEP 18618-000, Botucatu, SP, fone (0XX14) 6802-6053, eoono@ibb.unesp.br / mingo@ibb.unesp.br

Rev. Bras. Frutic., Jaboticabal - SP, v. 24, n. 2, p.306-311, agosto 2002 
O tamanho do fruto é importante não somente porque é um dos componentes do rendimento produtivo, mas também porque determina a aceitação do mesmo no mercado. Já a porcentagem de suco é um variável extremamente importante para o seu processamento industrial, estando também relacionada ao tamanho do fruto (Guardiola, 1992).

Desta maneira, o presente trabalho teve por objetivo avaliar os efeitos de alguns reguladores vegetais, do grupo das auxinas e giberelinas, combinados e aplicados em pré-colheita na queda natural dos frutos e sua influência no desenvolvimento final dos frutos de laranjeira- 'Hamlin'.

\section{MATERIAL E MÉTODOS}

O presente trabalho foi conduzido em condições de campo na Fazenda Olho d'Água, pertencente à Citrovita Agroindustrial Ltda., localizada no município de ItapetiningaSP, em 1993, sendo utilizadas árvores de laranja (Citrus sinensis Osbeck) cultivar Hamlin com quatro anos de idade. Durante o experimento, foram realizados todos os tratos culturais com relação à nutrição, controle de plantas daninhas, pragas e doenças.

Os tratamentos empregados foram: 1- $\mathrm{GA}_{3}$ (ácido giberélico) 12,5 + NAA (ácido naftaleno-acético) 12,5mg.L-1 ; 2$\mathrm{GA}_{3} 25$ + NAA 25mg.L-1 $; 3-\mathrm{GA}_{3} 12,5$ + 2,4-D (ácido 2,4diclorofenoxi-acético) 12,5mg. $\mathrm{L}^{-1} ; 4-\mathrm{GA}_{3} 25+2,4-\mathrm{D} 25 \mathrm{mg} . \mathrm{L}^{-1} ; 5-$

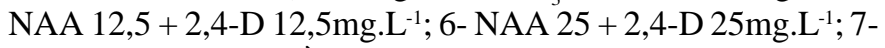
testemunha (água). À solução de tratamentos, adicionou-se espalhante adesivo Extravon a $0,3 \%$, contendo $250 \mathrm{~g} / \mathrm{l}$ de alquilfenol-poliglicoéter. Foram gastos $4 \mathrm{~L}$ de solução por planta com pulverizador do tipo PH600 da Jacto, acionado na tomada do trator, a pressão constante de 200 libras. Durante o período experimental, foram realizadas três aplicações, sendo a primeira realizada no dia 25-03, no início da virada de cor dos frutos, a segunda e a terceira a intervalos de 45 dias.

O delineamento experimental foi inteiramente casualizado, com seis plantas em linha por parcela, sete tratamentos, com seis repetições, totalizando 42 parcelas.

As variáveis avaliadas foram: massa fresca de frutos $(\mathrm{g})$, porcentagem de queda natural de frutos e teor de suco no fruto (\%). Essas variáveis foram avaliadas em oito colheitas, sendo a primeira realizada no dia 25-03 (antes da aplicação dos tratamentos) e as demais a intervalos de 30 dias entre cada colheita, sendo a última realizada no dia 29-10.

A porcentagem de queda natural foi obtida pela contagem semanal do número de frutos caídos sob a copa das plantas, sem a presença de picadas de insetos, por parcela de quatro plantas, adicionando-se em cada data o número de frutos caídos desde a última colheita, sendo expressos em porcentagem do total de frutos existentes em cada parcela. Mas, como os valores encontrados até a quarta colheita foram muito próximos de zero, esta variável foi analisada estatisticamente a partir da quinta colheita.

Para a determinação do teor de suco, foram subtraídos o peso fresco do fruto $(\mathrm{g})$ e o peso fresco do suco, extraído em extratora de copo, livre da casca, polpa e sementes, sendo os resultados expressos em porcentagem (Coelho \& Cunha, 1982). Em cada colheita, foram selecionados cinco frutos ao acaso, de cada uma das duas plantas, totalizando dez frutos por amostra.
Para a análise dos dados, utilizou-se o modelo linear multivariado, testando-se, conjuntamente, as diferenças para cada uma das variáveis estudadas. Para a realização das análises, utilizou-se o programa Perfil (Rosa, 1994), sendo testadas cinco hipóteses: $\mathrm{H}_{01}=$ os perfis dos sete grupos são paralelos, têm $\mathrm{o}$ mesmo comportamento; $\mathrm{H}_{02}=$ aceitando $\mathrm{H}_{01}$, não há diferenças entre os sete grupos, considerando o perfil global; $\mathrm{H}_{03}=$ aceitando $\mathrm{H}_{01}$, não há diferenças entre as quatro condições para o conjunto dos sete grupos; $\mathrm{H}_{04}=$ não há diferença entre as quatro condições dentro de cada grupo; $\mathrm{H}_{05}=$ análise de variância para verificar efeito de grupo dentro de cada condição. Em todas as análises estatísticas, o nível de probabilidade adotado foi de 5\%.

Como o número de repetições (seis) do experimento foi menor que o número de colheitas (oito), foi necessária, para a realização da análise estatística, a divisão do número de colheitas em dois períodos: $1^{\circ}$ período, referente às colheitas I, II, III e $\mathrm{IV}$, e o $2^{\circ}$ período, referente às colheitas V, VI, VII e VIII.

\section{RESULTADOS E DISCUSSÃO}

\section{Massa fresca de frutos}

A primeira hipótese testada na análise de perfil $\left(\mathrm{H}_{01}\right)$, referente ao paralelismo dos perfis dos sete tratamentos estudados, não foi rejeitada para esta variável, denotando comportamento similar dos tratamentos no decorrer do período experimental, ou, ainda, a ausência de interação entre tratamento e colheita. Uma vez satisfeita esta hipótese de paralelismo, procede-se com a verificação das hipóteses $\mathrm{H}_{02}$ e $\mathrm{H}_{03}$, para a comparação dos tratamentos e das colheitas (Tabela 1).

Para este caso, não se observa efeito significativo dos tratamentos, porém observa-se efeito significativo das datas de colheita. A partir das Tabelas 1 e 2, conclui-se que os ganhos médios em massa fresca, nos sete grupos estudados, apresentam perfis paralelos e coincidentes ( $p>0,05$ ), não existindo diferença significativa entre os mesmos.

Tais resultados diferem dos obtidos por Barros (1992), em tangerina-'Poncã', nos quais o tratamento com GA 3 20mg.L' ${ }^{1}+2,4-\mathrm{D} 20 \mathrm{mg}$.L $\mathrm{L}^{-1}$ aumentou a massa do fruto em relação à testemunha.

Segundo Garcia-Luíz et al. (1992), a massa fresca de frutos de tangerina- 'Clementina' cresce até um mês antes da época ideal de colheita determinada pelo "ratio", mas depois tende à estabilização, sendo que o $\mathrm{GA}_{3}$ não aumenta a massa fresca de frutos. Tais resultados dão suporte aos resultados obtidos para todos os tratamentos, já que os mesmos não mostraram efeitos positivos do $\mathrm{GA}_{3}$, do 2,4-D ou do NAA sobre a massa fresca dos frutos.

Porém, para datas de colheitas, observa-se acréscimo na massa fresca de frutos até a sexta colheita, havendo diferença estatística entre as colheitas I, II, III e IV e entre a V, VI e VII, não diferindo esta última da oitava colheita. Na colheita $\mathrm{V}$, houve queda acentuada na massa dos frutos. Esta queda deveu-se, possivelmente, à perda de água natural agravada pelo baixo índice pluviométrico no mês (Tabela 7). Por outro lado, a queda na massa fresca, verificada na colheita VII, deveu-se, possivelmente, à ocorrência da florada da safra seguinte.

\section{Teor de suco no fruto}


Também para esta variável, a primeira hipótese testada $\left(\mathrm{H}_{01}\right)$ referente ao paralelismo dos perfis não foi rejeitada, denotando comportamento similar dos tratamentos, porém nota-se efeito significativo das colheitas (Tabela 3 ).

A partir das Tabelas 3 e 4, pode-se verificar que os ganhos médios em teor de suco, nos sete grupos estudados, apresentam perfis paralelos e coincidentes ( $p>0,05$ ), não existindo diferença significativa entre os mesmos. Assim, a aplicação dos reguladores vegetais não favoreceu no aumento do teor de suco dos frutos.

Para as colheitas, ocorrem flutuações no teor de suco com o decorrer do tempo, aumentando até atingir o máximo de porcentagem de suco na quinta colheita, havendo diferença estatística entre estas e as demais, após a qual houve decréscimo na porcentagem de suco a partir da sexta colheita, com estas não diferindo entre si até a última colheita.

Também El-Otmani \& Coggins Jr. (1991), pulverizando plantas de laranja-'Washington Navel', com GA 3 10mg.L.' e 2,4D $16 \mathrm{mg} . \mathrm{L}^{-1}$, isolados ou em combinação, obtiveram maior porcentagem de suco.

Após alcançado o "ratio" ideal para a colheita, próximo à quarta colheita para todos os tratamentos, os frutos tenderam a perder água, visto que, a partir da sexta colheita, já tivera início a florada da safra seguinte, a qual coincidiu com o défice hídrico ocorrido nos meses de julho e agosto (Tabela 7). Para Agustí \& Almela (1991), a maturação dos frutos cítricos acha-se fortemente influenciada pelo clima; devendo-se considerar que a manutenção dos frutos na árvore, até sua senescência avançada, provoca perda de qualidade, pois o fruto segue crescendo, perden- do-se suco e açúcares, e a acidez cai a valores insípidos, inferiores a $0,5 \%$.

Os tratamentos com NAA diferiram da testemunha, mostrando a ausência de efeito desses tratamentos no teor de suco.

\section{Porcentagem de queda natural dos frutos}

Verificando-se a análise referente à variável porcentagem de queda natural, contida na Tabela 5, pode-se observar que, diferentemente dos resultados obtidos para as variáveis anteriores, a hipótese de paralelismo foi rejeitada, sugerindo interação entre tratamento e colheitas. Para esta situação, continuou-se a análise para verificar as hipótese $\mathrm{H}_{04}$ e $\mathrm{H}_{05}$, referentes ao estudo de condições das colheitas em cada tratamento e ao estudo de tratamentos em cada colheita.

Para este caso, pode-se verificar que, para a porcentagem de queda, os sete perfis estudados não são semelhantes $(\mathrm{p}<0,05)$. Com relação ao efeito de período em cada tratamento, pode-se verificar, pelas Tabelas 5 e 6 , que somente o perfil relativo à testemunha é crescente do primeiro ao terceiro período, não o sendo para os demais tratamentos.

A partir do terceiro período, nota-se que os perfis são crescentes para os tratamentos com GA $12,5+\mathrm{NAA} 12,5 \mathrm{mg} . \mathrm{L}^{-1}$, GA $_{3}$ 12,5+2,4-D 12,5mg.L.-1 e NAA 12,5+2,4-D 12,5mg.L $\mathrm{L}^{-1}$ e continuam não demonstrando crescimento para os tratamentos com $\mathrm{GA}_{3} 25$ + NAA $25 \mathrm{mg} . \mathrm{L}^{-1}, \mathrm{GA}_{3} 25$ + 2,4-D $25 \mathrm{mg} . \mathrm{L}^{-1}$ e NAA $25+2,4-$ D $25 \mathrm{mg} . \mathrm{L}^{-1}$. O perfil da testemunha continuou apresentando crescimento até o quarto período.

No tocante à comparação entre os tratamentos $\left(\mathrm{H}_{05}\right)$, verifica-se que somente o tratamento com GA $325+2,4-\mathrm{D} 25 \mathrm{mg} \cdot \mathrm{L}^{-1}$

TABELA 1 - Resultado da análise multivariada de perfis para peso fresco (g), dos frutos de laranjeira- 'Hamlin', submetidos a tratamentos com fitorreguladores, nas várias datas de colheita.

\begin{tabular}{lll}
\hline Hipótese estatística & resultado do teste estatístico & conclusão \\
\hline Paralelismo entre os perfis $\left(\mathrm{H}_{01}\right)$ & $\theta=0,279(\mathrm{p}>0,05)$ & existe semelhança entre os perfis \\
& $\theta=0,194(\mathrm{p}>0,05)$ & \\
\hline Coincidência entre os perfis $\left(\mathrm{H}_{02}\right)$ & $\mathrm{F}=1,370(\mathrm{p}>0,05)$ & $1=2=3=4=5=6=7$ \\
& $\mathrm{~F}=0,580(\mathrm{p}>0,05)$ & $1=2=4=5=6=7$ \\
\hline Efeito de período $\left(\mathrm{H}_{03}\right)$ & $\mathrm{F}=52,96(\mathrm{p}<0,05)$ & $\mathrm{I}<\mathrm{II}<\mathrm{III}<\mathrm{IV}$ \\
& $\mathrm{F}=8,46(\mathrm{p}<0,05)$ & $\mathrm{V}<\mathrm{VI}>$ VII $=$ VIII \\
\hline
\end{tabular}

TABELA 2 - Médias da massa fresca (g) de frutos de laranjeira- 'Hamlin', submetidos a tratamentos com fitorreguladores, nas oito datas de colheitas.

\begin{tabular}{|c|c|c|c|c|c|c|c|c|}
\hline Tratamentos & I & II & III & IV & $\mathrm{V}$ & VI & VII & VIII \\
\hline $\mathrm{GA}_{3}+\mathrm{NAA} 12,5 \mathrm{mg} \cdot \mathrm{L}^{-1}$ & 114,7 & 122,2 & 1393 & 149,0 & 139,0 & 158,3 & 147,3 & 154,0 \\
\hline $\mathrm{GA}_{3}+\mathrm{NAA} 25 \mathrm{mg} \cdot \mathrm{L}^{-1}$ & 115,7 & 126,1 & 136,7 & 144,0 & 140,5 & 163,7 & 148,0 & 155,3 \\
\hline $\mathrm{GA}_{3}+2,4-\mathrm{D} 12,5 \mathrm{mg} \cdot \mathrm{L}^{-1}$ & 124,7 & 134,2 & 137,7 & 152,7 & 145,0 & 166,7 & 157,3 & 156,1 \\
\hline $\mathrm{GA}_{3}+2,4-\mathrm{D} 25 \mathrm{mg} \cdot \mathrm{L}^{-1}$ & 127,3 & 128,3 & 135,0 & 152,3 & 155,0 & 168,0 & 153,7 & 152,3 \\
\hline $\mathrm{NAA}+2,4-\mathrm{D} 12,5 \mathrm{mg} \cdot \mathrm{L}^{-1}$ & 126,0 & 136,9 & 153,7 & 169,7 & 158,0 & 159,0 & 152,7 & 151,7 \\
\hline $\mathrm{NAA}+2,4-\mathrm{D} 25 \mathrm{mg} \cdot \mathrm{L}^{-1}$ & 127,7 & 137,8 & 136,3 & 159,3 & 145,0 & 166,7 & 159,3 & 148,0 \\
\hline testemunha (água) & 127,3 & 129,4 & 136,7 & 154,7 & 141,3 & 153,0 & 153,7 & 153,0 \\
\hline Médias & 123,3 & 130,7 & 139,3 & 154,5 & 146,3 & 162,2 & 153,1 & 152,9 \\
\hline
\end{tabular}

Rev. Bras. Frutic., Jaboticabal - SP, v. 24, n. 2, p.306-311, agosto 2002 
TABELA 3 - Resultado da análise multivariada de perfis para teor de suco (\%) dos frutos de laranjeira-'Hamlin', submetidos a tratamentos com fitorreguladores, nas várias datas de colheita.

\begin{tabular}{lll}
\hline Hipótese estatística & resultado do teste estatístico & conclusão \\
\hline Paralelismo entre os perfis $\left(\mathrm{H}_{01}\right)$ & $\theta=0,152(\mathrm{p}>0,05)$ & existe semelhança entre os perfis \\
& $\theta=0,257(\mathrm{p}>0,05)$ & \\
\hline Coincidência entre os perfis $\left(\mathrm{H}_{02}\right)$ & $\mathrm{F}=0,440(\mathrm{p}>0,05)$ & $1=2=3=4=5=6=7$ \\
& $\mathrm{~F}=2,320(\mathrm{p}>0,05)$ & $1=2=4=5=6=7$ \\
\hline Efeito de período $\left(\mathrm{H}_{03}\right)$ & $\mathrm{F}=133,58(\mathrm{p}<0,05)$ & $\mathrm{I}<\mathrm{II}=\mathrm{III}=\mathrm{IV}$ \\
& $\mathrm{F}=12,23(\mathrm{p}<0,05)$ & $\mathrm{V}>$ VI $=$ VII $=$ VIII
\end{tabular}

TABELA 4 - Médias do teor de suco (\%) dos frutos de laranjeira-'Hamlin', submetidos a tratamentos com fitorreguladores, nas oito datas de colheitas.

\begin{tabular}{|c|c|c|c|c|c|c|c|c|}
\hline Tratamentos & I & II & III & IV & $\mathrm{V}$ & VI & V II & V III \\
\hline $\mathrm{GA}_{3}+\mathrm{NAA} 12,5 \mathrm{mg} \cdot \mathrm{L}^{-1}$ & 41,94 & 50,96 & 49,07 & 50,35 & 54,82 & 49,58 & 51,03 & 53,31 \\
\hline $\mathrm{GA}_{3}+\mathrm{NAA} 25 \mathrm{mg} . \mathrm{L}^{-1}$ & 41,58 & 51,94 & 51,77 & 51,40 & 53,32 & 51,71 & 50,38 & 48,39 \\
\hline $\mathrm{GA}_{3}+2,4-\mathrm{D} 12,5 \mathrm{mg} \cdot \mathrm{L}^{-1}$ & 41,18 & 50,90 & 48,43 & 52,09 & 54,14 & 50,80 & 49,45 & 50,82 \\
\hline $\mathrm{GA}_{3}+2,4-\mathrm{D} 25 \mathrm{mg} \cdot \mathrm{L}^{-1}$ & 41,73 & 49,74 & 51,19 & 52,05 & 52,81 & 50,60 & 50,95 & 50,23 \\
\hline $\mathrm{NAA}+2,4-\mathrm{D} 12,5 \mathrm{mg} \cdot \mathrm{L}^{-1}$ & 43,11 & 51,45 & 48,83 & 51,78 & 52,52 & 48,19 & 46,62 & 47,22 \\
\hline $\mathrm{NAA}+2,4-\mathrm{D} 25 \mathrm{mg} \cdot \mathrm{L}^{-1}$ & 41,34 & 49,07 & 50,75 & 51,18 & 52,53 & 48,55 & 48,80 & 49,89 \\
\hline testemunha (água) & 41,14 & 49,29 & 51,55 & 51,91 & 53,40 & 49,97 & 51,16 & 50,30 \\
\hline Médias & 41,72 & 50,48 & 50,23 & 51,54 & 53,36 & 49,41 & 49,77 & 50,02 \\
\hline
\end{tabular}

TABELA 5 - Resultado da análise multivariada de perfis para taxa de queda natural (\%) dos frutos de laranjeira-'Hamlin', submetidos a tratamentos com fitorreguladores, nas várias datas de colheita.

\begin{tabular}{lll}
\hline Hipótese estatística & resultado do teste estatístico & Conclusão \\
\hline Paralelismo entre os perfis $\left(\mathrm{H}_{01}\right)$ & $\theta=0,825(\mathrm{p}<0,05)$ & não existe semelhança entre os perfis \\
\hline Efeito de período em cada tratamento $\left(\mathrm{H}_{04}\right)$ & $1: \mathrm{F}=11,93(\mathrm{p}<0,05)$ & $\mathrm{I}=\mathrm{II}=\mathrm{III}<\mathrm{IV} ; \mathrm{I}<\mathrm{IV} ; \mathrm{II}<\mathrm{IV}$ \\
& $2: \mathrm{F}=4,50(\mathrm{p}>0,05)$ & $\mathrm{I}=\mathrm{II}=\mathrm{III}=\mathrm{IV}$ \\
& $3: \mathrm{F}=8,88(\mathrm{p}<0,05)$ & $\mathrm{I}=\mathrm{II}=\mathrm{III}<\mathrm{IV}$ \\
& $4: \mathrm{F}=1,32(\mathrm{p}>0,05)$ & $\mathrm{I}=\mathrm{II}=\mathrm{III}=\mathrm{IV}$ \\
& $5: \mathrm{F}=12,54(\mathrm{p}<0,05)$ & $\mathrm{I}=\mathrm{II}=\mathrm{III}<\mathrm{IV} ; \mathrm{I}<\mathrm{IV} ; \mathrm{II}<\mathrm{IV}$ \\
& $6: \mathrm{F}=3,65(\mathrm{p}>0,05)$ & $\mathrm{I}=\mathrm{II}=\mathrm{III}=\mathrm{IV}$ \\
& $7: \mathrm{F}=47,47(\mathrm{p}<0,05)$ & $\mathrm{I}<\mathrm{II}<\mathrm{III}<\mathrm{IV}$ \\
\hline Efeito de tratamento em cada período $\left(\mathrm{H}_{05}\right)$ & $\mathrm{I}: \mathrm{F}=3,71(\mathrm{p}<0,05)$ & $4<7$ \\
& $\mathrm{II}: \mathrm{F}=8,33(\mathrm{p}<0,05)$ & $2<7 ; 3<7 ; 4<7 ; 5<7 ; 6<7$ \\
& $\mathrm{III}: \mathrm{F}=23,92(\mathrm{p}<0,05)$ & $(1=2=3=4=5=6)<7$ \\
& $\mathrm{IV}: \mathrm{F}=20,34(\mathrm{p}<0,05)$ & $(1=2=3=4=5=6)<7$ \\
\hline
\end{tabular}

TABELA 6 - Médias da taxa de queda natural (\%) dos frutos de laranjeira-'Hamlin', submetidos a tratamentos com fitorreguladores, nas várias datas de colheitas.

\begin{tabular}{|c|c|c|c|c|}
\hline Tratamentos & $\mathrm{V}$ & V I & V II & V III \\
\hline $\mathrm{GA}_{3}+\mathrm{NAA} 12,5 \mathrm{mg} \cdot \mathrm{L}^{-1}$ & 0,77 & 3,12 & 14,15 & 25,26 \\
\hline $\mathrm{GA}_{3}+\mathrm{NAA} 25 \mathrm{mg} \cdot \mathrm{L}^{-1}$ & 1,42 & 1,06 & 4,03 & 10,57 \\
\hline $\mathrm{GA}_{3}+2,4-\mathrm{D} 12,5 \mathrm{mg} \cdot \mathrm{L}^{-1}$ & 0,43 & 0,98 & 5,24 & 14,59 \\
\hline $\mathrm{GA}_{3}+2,4-\mathrm{D} 25 \mathrm{mg} \cdot \mathrm{L}^{-1}$ & 0,09 & 0,26 & 0,91 & 4,17 \\
\hline $\mathrm{NAA}+2,4-\mathrm{D} 12,5 \mathrm{mg} \cdot \mathrm{L}^{-1}$ & 0,81 & 2,35 & 11,73 & 23,41 \\
\hline $\mathrm{NAA}+2,4-\mathrm{D} 25 \mathrm{mg} \cdot \mathrm{L}^{-1}$ & 0,56 & 1,05 & 4,74 & 10,92 \\
\hline testemunha (água) & 0,98 & 6,13 & 39,13 & 54,03 \\
\hline
\end{tabular}


TABELA 7 - Precipitação (mm) e temperatura $\left({ }^{\circ} \mathrm{C}\right)$ ocorridas na área experimental, no ano de 1993.

\begin{tabular}{lcccc}
\hline Meses & Temp. média & Temp. mínima & Temp. máxima & Precipitação \\
\hline Janeiro & 23,09 & 18,56 & 29,84 & 203,20 \\
Fevereiro & 21,17 & 17,88 & 26,61 & 194,60 \\
Março & 22,33 & 18,01 & 29,12 & 126,90 \\
Abril & 21,03 & 16,06 & 28,08 & 47,70 \\
Maio & 18,01 & 13,02 & 24,71 & 101,30 \\
Junho & 15,04 & 9,54 & 22,21 & 259,93 \\
Julho & 9,32 & 22,75 & 31,10 \\
Agosto & 15,25 & 8,25 & 22,98 & 29,40 \\
Setembro & 14,68 & 11,47 & 23,09 & 159,10 \\
Outubro & 16,45 & 15,29 & 27,34 & 79,60 \\
Novembro & 20,82 & 16,49 & 29,86 & 56,40 \\
Dezembro & 22,73 & 18,31 & 29,53 & 58,40 \\
\hline
\end{tabular}

apresentou porcentagem de queda inferior à testemunha a partir do primeiro período de avaliação. Na Tabela 6 , verifica-se que, no segundo período de avaliação, somente o tratamento com $\mathrm{GA}_{3}$ 12,5+ NAA 12,5mg.L-1 , foi semelhante à testemunha, sendo que os demais tratamentos apresentaram porcentagem de queda inferior ao mesmo. A partir do terceiro período, todos os tratamentos apresentaram porcentagem de queda natural significativamente inferior à testemunha $(\mathrm{p}<0,05)$.

Segundo Monselise (1979), o 2,4-D é uma auxina sintética frequientemente utilizada nas doses de 5 a $20 \mathrm{mg} . \mathrm{L}^{-1}$, como antagonista da abscisão de frutos maduros em praticamente todas as espécies cítricas, diminuindo a atividade da celulase e da poligalacturonase, inibindo a separação do cálice do fruto.

El-Otmani et al.(1990), pulverizando quatro diferentes cultivares de citros, com 10mg. $\mathrm{L}^{-1}$ de GA 3 e $16 \mathrm{mg} . \mathrm{L}^{-1}$ de 2,4-D, combinados ou isolados, relatam que as maiores porcentagens de queda ocorreram em plantas não tratadas com tais reguladores vegetais, $\mathrm{e}$ as menores porcentagens ocorreram nos tratamentos onde o 2,4-D foi aplicado isoladamente.

Pode-se observar, pela Tabela 6, que todos os tratamentos contendo os reguladores vegetais foram efetivos na redução da queda pré-colheita, embora em diferentes níveis. GA 12,5 +NAA $12,5 \mathrm{mg}$. $\mathrm{L}^{-1}$ reduziu a queda natural da ordem de $53,49 \%$; NAA $12,5+2,4-D$ 12,5mg. $\mathrm{L}^{-1}$ reduziu a queda a 56,89\% em média; com GA 3 12,5+2,4-D 12,5mg. $\mathrm{L}^{-1}$, a redução encontrada foi da ordem de 73,13\%; para NAA 25+2,4-D 25mg.L-1 , a redução de queda foi de 79,88\%; com GA 325 + NAA $25 \mathrm{mg} . \mathrm{L}^{-1}$, a redução encontrada foi de $80,54 \%$ e, finalmente, obteve-se redução na queda natural de $92,32 \%$ para o tratamento com $\mathrm{GA}_{3} 25+2,4-\mathrm{D}$ $25 \mathrm{mg}$. $\mathrm{L}^{-1}$. Embora não ocorrendo diferença significativa entre os tratamentos com reguladores vegetais, as maiores reduções na queda dos frutos da laranjeira- 'Hamlin' foram obtidas nos tratamentos que apresentaram as maiores concentrações $\left(25 \mathrm{mg} . \mathrm{L}^{-1}\right)$ dos reguladores vegetais utilizados.

Esta dependência da abscisão ao conteúdo endógeno de auxinas tem sido provada mediante aplicações exógenas de 2,4D ou NAA, visto que o transporte das auxinas pela planta perdura durante muito tempo sem que o etileno pareça afetá-lo
(Agustí \& Almela, 1991).

Tais fatos poderiam explicar a baixa porcentagem de queda também encontrada no tratamento com GA $325+$ NAA $25 \mathrm{mg} . \mathrm{L}^{-}$ ${ }^{1}$, devendo-se, desta forma, a redução da queda dos frutos à interação destes dois reguladores vegetais, os quais se mostram tão eficazes quanto os tratamentos com 2,4-D. Da mesma maneira, a baixa porcentagem de queda obtida no tratamento com NAA $25+2,4$-D 25mg.L-1 deveu-se, possivelmente, à ação conjunta destas duas auxinas.

\section{CONCLUSÃO}

Nas condições estudadas e com base nos resultados obtidos, pode-se concluir que a utilização de reguladores vegetais, para proporcionar o desenvolvimento dos frutos, não influenciou no crescimento e no teor de suco dos frutos de laranjeira'Hamlin', mas reduziu a queda natural de frutos de laranjeira'Hamlin', destacando-se o tratamento com GA 3 25mg.L-1 $+2,4-\mathrm{D}$ 25mg.L $\mathrm{L}^{-1}$.

\section{REFERÊNCIAS BIBLIOGRÁFICAS}

AGUSTÍ, M.; ALMELA, V. Aplicación de fitorreguladores en citricultura. Barcelona: AEDOS, 1991. 261p.

ARANGUREN M.; ALFARO, D.; CASTRO, Y.O. Efecto de aplicaciones precosecha de 2,4-D sobre el desarrollo del fruto y el control de la caídas por rajaduras em naranja 'Valência'. Ciênc. Tec. Agric. Cítricos Otros Frutales, v.11, n.3, p.113-120, 1988.

BARROS, S.A. Efeitos da aplicação pré-colheita de fitorreguladores, no desenvolvimento final e maturação do fruto da tangerineira 'Ponkan' (Citrus reticulata Blanco). 1992. 105f. Dissertação (Mestrado) - Faculdade de Ciências Agrárias, Universidade Estadual Paulista, Botucatu, 1992. 
CHITARRA, M.I.F.; CHITARRA, A.B. Pós-colheita de frutos e hortaliças: fisiologia e manuseio. Lavras: ESAL/Fundação de Apoio ao Ensino, Pesquisa e Extensão, 1990. 293p.

COELHO, Y.S.; CUNHA, G.A. Critérios de avaliação da maturação e qualidade de frutos, com ênfase para citros e abacaxi. Circular Técnica Centro Nacional Pesquisa da Mandioca e Fruticultura, Cruz das Almas, v.1, p.1-20, 1982.

EL-OTMANI, M.; BAREK, A.A.; COGGINS JUNIOR, C.W. GA and 2,4-D prolong on tree storage of citrus in Morocco. Scientia Horticulturae, Amsterdan, v.44, p.241-249, 1990.

EL-OTMANI, M.; COGGINS JUNIOR, C.W. Growth regulator effects on retention of quality of stored citrus fruit. Scientia Horticulturae, Amsterdan, v.45, p.261-272, 1991.

EL-ZEFTAWI, B.M. Effects of gibberellic acid and cycocel on colouring and sizing of lemon. Scientia Horticulturae, Amsterdan, v.12, p.177-181, 1980.

GARCIA-LUÍZ, A.; HERRERO-VILLEN, A.; GUARDIOLA, J.L. Effects of applications of gibberellic acid on late growth, maturation and pigmentation of the Clementine mandarin. Scientia Horticulturae, Amsterdan, v.49, n.1-2, p.71-82, 1992.

GUARDIOLA, J.L. Frutificação e crescimento. In: SEMINÁRIO INTERNACIONAL DE CITROS -FISIOLOGIA, 2., 1992. Bebedouro. Anais ... Campinas: Fundação Cargill, 1992. p.1-26.
LLANO, J.L.; BETANCOURT, M., DIAZ, J. Inhibition of mature grapefruit drop. Use of growth regulators. Cultivos Industriales, n.2-3, p.17-26, 1991.

MONSELISE, S.P. The use of growth regulators in citriculture: a review. Scientia Horticulturae, Amsterdan, v.11, p.151-162, 1979.

MONSELISE, S.P.; GOREN, R. The role of internal factors and exogenous control in flowering, peel growth, and abscission in citrus. Hortscience, Alexandria, v.13, n.2, p.134-139, 1978.

MORRISON, D.F. Multivariate statistical methods. New York: McGraw-Hill, 1967. 415p.

PATIL, V.S.; KEDR, V.P.; NAGRE, P.K. Effect of foliar sprays of plant growth regulators and urea on premature and preharvest fruit drop in 'Kinnow' mandarin. PKV Res. J., v.13, n.2, p.161$163,1989$.

RAGONE, M.L. Os reguladores de crescimento no cultivo cítrico na Argentina. In: SEMINÁRIO INTERNACIONAL DE CITROS, 1992, Bebedouro, SP. Anais ... Campinas: Fundação Cargill, 1992. p.52-66.

ROSA, G.J.M. Desenvolvimento de aplicativo para a análise de perfil na experimentação zootécnica. 72f. Dissertação (Mestrado) - Faculdade de Medicina, Veterinária e Zootecnia, Universidade Estadual Paulista, Botucatu,1994. 